\title{
EXISTENCE AND UNIQUENESS OF SOLUTIONS OF SCHRÖDINGER TYPE STATIONARY EQUATIONS WITH VERY SINGULAR POTENTIALS WITHOUT PRESCRIBING BOUNDARY CONDITIONS AND SOME APPLICATIONS
}

\author{
JESÚS ILDEFONSO DÍAZ, DAVID GÓMEZ-CASTRO AND \\ JEAN MiCHEL RAKOTOSON
}

\begin{abstract}
Motivated mainly by the localization over an open bounded set $\Omega$ of $\mathbb{R}^{n}$ of solutions of the Schrödinger equations, we consider the Schrödinger equation over $\Omega$ with a very singular potential $V(x) \geqslant C d(x, \partial \Omega)^{-r}$ with $r \geqslant 2$ and a convective flow $\vec{U}$. We prove the existence and uniqueness of a very weak solution of the equation, when the right hand side datum $f(x)$ is in $L^{1}(\Omega, d(\cdot, \partial \Omega))$, even if no boundary condition is a priori prescribed. We prove that, in fact, the solution necessarily satisfies (in a suitable way) the Dirichlet condition $u=0$ on $\partial \Omega$. These results improve some of the results of the previous paper by the authors in collaboration with Roger Temam. In addition, we prove some new results dealing with the $m$-accretivity in $L^{1}\left(\Omega, d(\cdot, \partial \Omega)^{\alpha}\right)$, where $\alpha \in[0,1]$, of the associated operator, the corresponding parabolic problem and the study of the complex evolution Schrödinger equation in $\mathbb{R}^{n}$.
\end{abstract}

Mathematics subject classification (2010): 35J75, 35J15, 35J25, 34K30, 76M23.

Keywords and phrases: Schrödinger equation, very singular potential, no boundary conditions, very weak distributional solution, local Kato inequality, accretive operator, complex evolution equation.

\section{REFERENCES}

[1] V. Barbu, Nonlinear Differential Equations of Monotone Types in Banach Spaces, Springer New York, New York, NY, 2010. doi:10.1007/978-1-4419-5542-5.

[2] C. Bennet, R. Sharpley, Interpolation of Operators. Academic Press, Boston (1988)

[3] Ph. Bénilan, L. Boccardo, Th. Gallouët, R. Gariepy, M. Pierre, J.L. Vázquez, An $L^{1}$-theory of existence and uniqueness of solutions of nonlinear elliptic equations, Ann. Scuala Norms Sup. Pisa, 22 (1995) 241-273.

[4] H. Brézis, Functional Analysis, Sobolev Spaces and Partial Differential Equations, Springer, New York, 2011.

[5] H. Brezis and T. Cazenave, Linear semigroups of contractions: the Hille-Yosida theory and some applications. Publications du Laboratoire d'Analyse Numérique, Université Pierre et Marie Curie, Paris, 1993.

[6] H. Brézis and T. Cazenave.: A Nonlinear Heat Equation with Singular Initial Data. J. d'Analyse Mathématique. 68, 277-304 (1996).

[7] H. Brézis and W.A. Strauss, Semi-linear second-order elliptic equations in $L^{1}$, J. Math. Soc. Japan. 25 (1973), 565-590.

[8] X. Cabré and Y. Martel, Existence versus explosion instantanée pour des équations de la chaleur linéaires avec potentiel singulier, Comptes Rendus l'Académie des Sci. - Ser. I - Math. 329 (1999) 973-978. doi:10.1016/S0764-4442(00)88588-2.

[9] M.G. Crandall and T.M. Liggett, Generation of Semi-Groups of Nonlinear Transformations on General Banach Spaces, American Journal of Mathematics. 93 No. 2 (1971) 265-298. 
[10] M. Fila, P. Souplet and F.B. Weissler, Linear and nonlinear heat equations in $L_{\delta}^{q}$ spaces and universal bounds for global solutions. Mathematische Annalen 113 (2001), 87-113. doi: $10.1007 / \mathrm{s} 002080100186$

[11] W.M. Frank and D.J. Land, Singular potentials. Rev. Mod. Phys. 43(1) (1971) 36-98.

[12] D. Daners and P. Koch Medina, Abstract evolution equations, periodic problems and applications, Longman, Harlow, 1992.

[13] J.I. Díaz, On the ambiguous treatment of Schrödinger equations for the infinite potential welland an alternative via flat solutions: The one-dimensional case. Interfaces and Free Boundaries 173 (2015) 333-351.

[14] J.I. Díaz, On the ambiguous treatment of the Schrödinger equation for the infinite potentialwell and an alternative via singular potentials: the multi-dimensional case. SeMA-Journal 743 (2017) 225-278, DOI 10.1007/s40324-017-0115-3.

[15] J. I. Díaz, D. Gómez-Castro, J.M. Rakotoson and R. Temam, Linear diffusion with singular absorption potential and/or unbounded convective flow: the weighted space approach. Discrete and Continuous Dynamical Systems. Volume 38, Number 2 (2018), 509-546.

[16] J.I. Díaz and J.-M. Rakotoson, Elliptic Problems on the Space of Weighted With the Distance To the Boundary Integrable Functions Revisited. Electron. J. Differ. Equations Conf. 21 (2012), 45-59.

[17] P. Drábek and J. Hernández, Quasilinear eigenvalue problems with singular weights for the $p$ Laplacian. To appear.

[18] D. Gilbarg and S. Trudinger, Elliptic partial differential equations of second order, Springer, Berlin 2001.

[19] D. Gómez-Castro, Homogenization and Shape Differentiation of Quasilinear Elliptic Problems. Applications to Chemical Engineering and Nanotechnology. Thesis at the UCM. 2017.

[20] A.D. Ionescu and C. E. Kenig, Uniqueness properties of solutions of Schrödinger equations, J. Funct. Anal. 232 (2006), no. 1, 90-136., https://doi.org/10.1016/j.jfa.2005.06.005 i

[21] J.M. Rakotoson, Linear equations with variable coefficients and Banach function spaces To appear.

[22] J.M. Rakotoson, Regularity of a very weak solution for parabolic equations and applications Advances in Differential Equations 16 (2011) 867-894.

[23] J.M. Rakotoson, New Hardy inequalities and behaviour of linear elliptic equations Journal of Functional Analysis 263 (2012) 2893-2920.

[24] M. Reed and B. Simon, Methods of Modern Mathematical Physics, Vol. II, Academic Press, New York 1975.

[25] L. Orsina and A. Ponce, Hopf potentials for the Schrödinger operator (version of 12 April 2017). 